\title{
Body Weight Gain Character Result in
} Standard Format

National Cancer Institute

\section{Source}

National Cancer Institute. Body Weight Gain Character Result in Standard Format. NCI

Thesaurus. Code C119774.

The standard character or string for representation and reporting of body weight gain data. 